\title{
El sistema constructivo empleado para la cimentación por pilotaje en las obras portuarias mediterráneas del siglo XVIII. El Real Arsenal de Cartagena
}

\author{
María Jesús Peñalver Martínez ${ }^{\mathrm{a}}$, Juan Francisco Maciá Sánchez ${ }^{\mathrm{a}}$ \\ aUniversidad Politécnica de Cartagena, Cartagena, España, mjesus.penalver@upct.es, juanfco.macia@upct.es
}

\begin{abstract}
The building works which presented the greatest technical and human challenge among all of those carried out during the construction of the Arsenal of Cartagena in 18th century are those of the quay surrounding the artificial dock. What is most complex and interesting about the construction of this structural element is, among other things, the depth at which cement was to be laid under the sea, the nature of the ground and the relief of the location. The structural solution for resolving this challenge evolved over the course of almost a century, as building knowledge, the skills of the head engineers and knowledge of the surrounding area increased. Several systems were proposed for laying the cement, all of which deserve particular attention. This article is based on the analysis of different historical dossiers found in the Simancas General Archive (Valladolid, Spain), containing manuscripts written by the architects responsible for the building work.
\end{abstract}

Keywords: underwater construction, foundations, constructive procces, s. XVIII.

\section{Introducción}

Las obras de construcción del muelle de la dársena del Real Arsenal de Cartagena darán comienzo del 20 de mayo de 1750. La expectación creada y la singularidad de estas obras queda reflejada en la siguiente reflexión del Ingeniero Director de las mismas, D. Sebastián Feringán Cortés

[...]. Las obras que seban a emprehender en este Puerto, creo no aya otras iguales: se ben estos casos rara vez: hize juicio que muchos por aprehender, solizitaran asistir a ellas, pero experimento al contrario [...]

La construcción del muelle comienza desde los ángulos del norte del rectángulo. Una vez abierta la obra en los vértices, se avanzará desde allí siguiendo las direcciones de los ejes cartesianos. De esta forma se simultanean las obras que se realizan en dirección norte-sur en las bandas laterales y las de este-oeste en la banda norte de la dársena.

Las variables que Feringán contempla para definir la solución constructiva definitiva son aquellas que garanticen la solidez y la permanencia de la obra a lo largo del tiempo, así como las que permitan la funcionalidad del muelle construido.

La solidez y permanencia del muelle requiere del correcto diseño del sistema de cimentación acorde con la naturaleza del terreno, de la acertada elección de materiales en función del medio donde se ubican y de la definición de los procesos adecuados para su correcta puesta en obra. La funcionalidad que se persigue es permitir el correcto embarco y desembarco de pertrechos y mercancías de las naves para las cuales se diseña el arsenal. Para ello, la solución 
que finalmente se ejecute deberá posibilitar la creación de una dársena con la profundidad necesaria para el calado de las mismas y una geometría que permita el correcto acercamiento de las embarcaciones para las operaciones de carga y descarga.

Por tanto, la solidez de la obra deteminará la definición del sistema de cimentación y su sistema de puesta en obra y su funcionalidad establecerá la profundidad de cimentación necesaria para construir el revestimiento del muelle.

En consecuencia, el modelo teórico inicialmente propuesto por Feringán para la construcción del muelle se deberá ir adaptando a las particularidades geotécnicas del terreno donde se ha de cimentar a medida que se tiene conocimiento de ellas en el trascurso de las obras.

Inicialmente, Feringán tenía previsto cimentar a 30 pies reales $(9,75 \mathrm{~m})$ de profundidad mediante una zapata corrida apoyada directamente en el fondo de la excavación, tal y como representó en su última propuesta realizada en diciembre de 1749 .

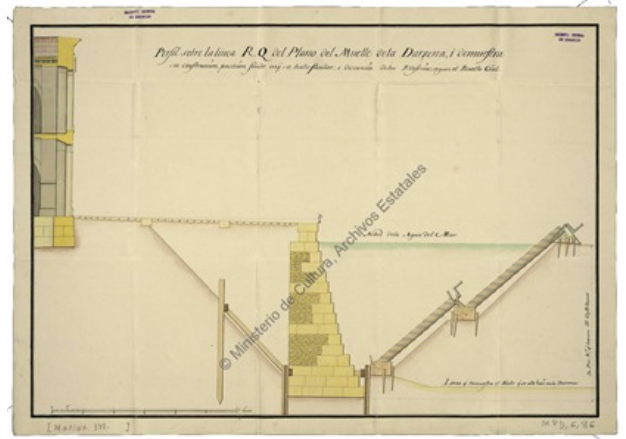

Fig. 1- Sección muelle (Feringán, 2/12/1749). A.G.S., MPD 06, 086 (Marina, Leg. 377). Ministerio de Cultura. Archivo General de Simancas

La aparición de un caudaloso venero en las excavaciones del ángulo noreste del muelle hace patente la imposibilidad de cimentar el muro con la solución prevista y la necesidad de utilizar en determinadas zonas una cimentación profunda mediante pilotaje.
La heterogeneidad de las características geomecánicas del terreno hace que la solución para cimentar el muelle no sea única en todo su perímetro. Ésta se adapta a la naturaleza del terreno que las obras en ejecución van evidenciando. En la imagen siguiente se representan en color amarillos las zonas de muelle finalmente cimentadas directamente sobre el fondo y en color naranja, aquellas en las que se hace necesaria la utilización de una cimentación mediante pilotes.

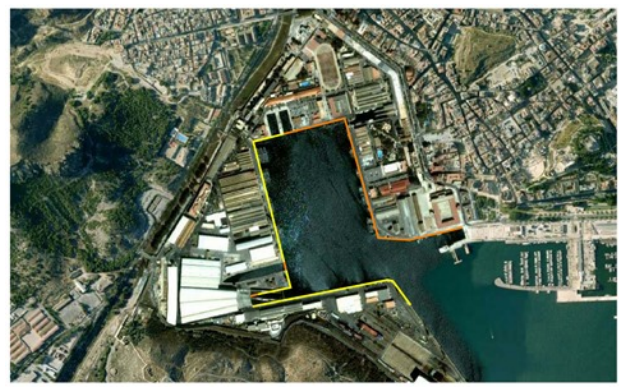

Fig. 2- Tipos de cimentación empleados en la construcción del muelle de la dársena de Cartagena (Peñalver, 2012)

\section{La configuración de la cimentación por pilotaje}

Las secciones transversales del muelle cimentado con pilotes difieren de unas bandas a otras e incluso lo hacen en el mismo desarrollo de éstas debido a las diferentes profundidades del plano de apoyo de la fábrica de sillería y al nivel del estrato de empotramiento de las puntas de los pilotes. Más allá de estas diferencias en las cotas de apoyo del muelle, la cimentación entendida como uno de los sistemas que componen su cortina, consta básicamente de los mismos elementos aunque las dimensiones de éstos se vayan adaptando sensiblemente a los condicionantes del entorno.

Los subsistemas que configuran la cimentación profunda utilizada son tres: el tablestacado o "palplanchado", los pilotes o "estacas" y el enrejado o "embarengado" (Fig. 3). Las funciones de cada uno de éstos son descritas por Feringán mediante bellas metáforas en el texto que se transcribe a continuación. 
[...]. A el mas empinado monte de estos contornos puede servir de permanente base laque el Muelle tiene: este es monte de peña artificial, y la virtud de ella adaptada al material, y comunicándose, reziprocamente de una a otra, sera toda peña unida y como de una pieza; con que una vez que aiga hecho cuerpo deque no dudan los Phissicos, es imposible, en lo natural, que ni la fuerza de las aguas superficiales le contraste, ni la blanda de los Veneros le perjudique, porque en el arte no cabe mas precauzion para el todo, que la que se a observado para la construcción de esta obra, pues para sentarla esta sobre un monte de Madera, y para resguardarla de las aguas

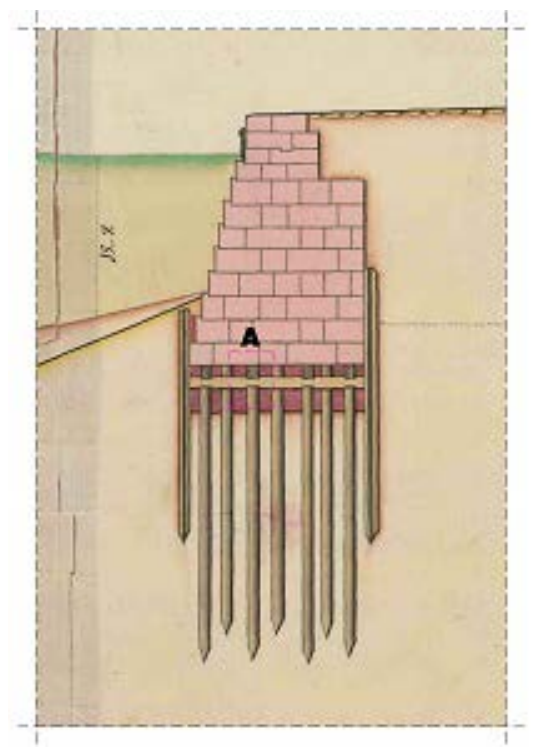

orizontales, tiene una Pinada de dos líneas de pared para dentro y fuera, en el Palplanchado que con sus engargolados de estaca y tablón alternativamente embarazan visiblemente la transpirazion de las aguas, [...].

La construcción de este sistema de cimentación se puede dividir en tres fases: la Fase 1, que comprende la ejecución del tablestacado que contendrá las paredes de la excavación en sus cotas más profundas y el clavado de los pilotes, la Fase 2, en la que se ejecuta el enrejado que arriostra las cabezas de las estacas y la Fase 3, consistente en el macizado de la retícula anterior de manera que se constituya una base firme y nivelada para el apoyo de la sillería.

Fig. 3- Elementos de la cimentación por pilotaje (Peñalver, 2012)

\subsection{El tablestacado y las máquinas de hinca}

El tablestacado utilizado en las obras del muelle puede ser analizado bajo dos perspectivas diferentes; como medio auxiliar empleado para posibilitar su ejecución o como parte integrante de su cimentación. Como estructura de contención permite realizar la excavación a la profundidad requerida y reduce el aflujo de aguas a la zanja. Como parte integrante del cimiento, a modo de bloque de guarda, garantiza la protección del cimiento y de las hiladas de sillares inferiores frente a las posibles agresiones causadas por las filtraciones de agua y los embates del mar. Este será el motivo de que una vez acabada la obra no se produzca su retirada.

El comportamiento adecuado de esta estructura de contención es fundamental en el proceso constructivo pues de él depende la profundidad a la que se podrá excavar para fundar la obra. Debe tener un diseño tal que le permita resistir los empujes del terreno sin excesivas deformaciones para garantizar la seguridad en las obras y una estanqueidad que dificulte la transpiración de aguas a la excavación de 
manera que puedan ser controladas con las bombas de achique manuales disponibles y así permitir clavar los pilotes y sentar la sillería.

Para conseguirlo, el ingeniero idea un ingenioso sistema de tablestacado mediante la construcción de una pared de piezas machiembradas de pino. En ella, se combinan estacas de sección cuadrada engargoladas en sus laterales que sirven de guía para el clavado de unos tablones de sección rectangular que se encajonan entre aquellas (Fig. 4). La sección de las primeras dota al conjunto de la resistencia y rigidez suficientes y el sistema de engarce entre éstas y los tablones de la impermeabilidad necesaria para poder obrar en su interior.

[...] dos lineas de pared para dentro y fuera, en el Palplanchado que con sus engargolados de estaca y tablon alternatibamente embarazan vissiblemente la transpirazion de las aguas que solo se experimenta por donde el acasso de un embarazo subterraneo desgobernó una estaca o tablon al tiempo de clavarle [...].

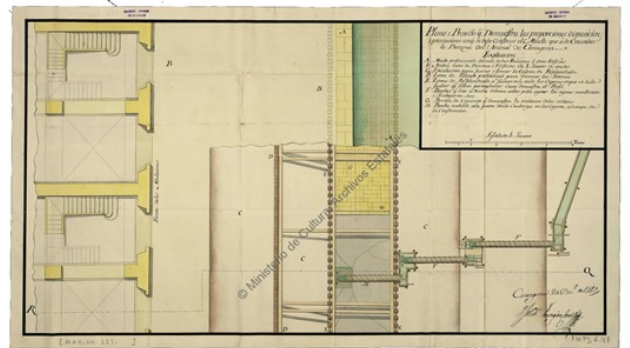

Fig. 4- Sistemas de contención y achique (Feringán, 2/12/1749). A.G.S., MPD 06, 087 (Marina, Leg. 377). Ministerio de Cultura. Archivo General de Simancas

Esta tipología de ataguías para la construcción de recintos tablestacados es mencionada en numerosos manuscritos y tratados anteriores (VV.AA, 1985). Cabe destacar el tablestacado propuesto para fundar dentro del agua en "Los veintiún libros de los ingenios y las máquinas” (Fig. 5).

Feringán adapta esta estructura a las condiciones de su obra. El ingeniero disminuye la distancia entre estacas engargoladas de manera que pueda cubrir este espacio con un solo tablón. Con ello consigue una doble finalidad; por un lado, evita la necesidad de calafatear la superficie interior de las ataguías cuando éstas se realizan mediante la adición de tablas y por otro, posibilita el hincado de la tabla a la profundidad que necesita.

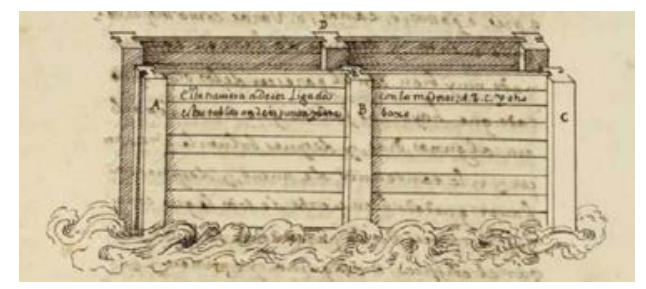

Fig. 5- Tablestacado para fundar dentro del agua (Turriano, s. XVI)

El ingeniero también debe mejorar los martinetes que se utilizan para el clavado de las estacas y tablones que componen esta estructura de contención. La profundidad a la que se deben clavar y la resistencia que ofrece el terreno en la mayoría de los casos, hace necesario la construcción de máquinas que lo posibiliten.

\section{[...] pues suspendida la Maza en 36 pies Reales} que hacen 42 castellanos de altura y dejada caer, a la estaca media pulgada la haze caminar a lo ultimo; y desde que a tablón y estaca les quedan seis pies que entran empiezan a rebatir la maza con 1050 libras (483 Kg) de pesso, que no la abra mayor, pues todas las que beemos practicadas, y escritas nolas proponen de 500 (230 Kg) a 600 libras (276 Kg) [...]. La primera se a hecho, con las proporziones que he dado, $y$ assistiendo asu disposizion, ha salido muy azertada, fuerte y de comodo manejo sin embargo que pessa quarenta y dos arrobas (483 $\mathrm{Kg}$ ); a lo que contribuie una grande roldana de Guayacan con exe de fierro, y sobre muñones de bronze, con fazilidad a mudarse, sin detener el trabajo, casso que con el usso se deteriore y con 18 peones sube la maza con altura y campaneo bastante para que el golpe sea violento, [...].

\subsection{Los pilotes y el enrejado}

Una vez concluido el tablestacado del fondo de la excavación y vaciado el terreno comprendido entre ambas líneas, se procede al hincado de las estacas de madera encargadas de transmitir las cargas a los estratos competentes donde las 
aguas impiden llegar mediante medios convencionales. Una vez descubiertas las cabezas de los mismos se fabricará sobre ellas el enrejado de madera que una vez macizado, dará unidad al conjunto y servirá como plano de apoyo a la fábrica de sillería.

Los pilotes se realizan con madera de pino y de sección cuadrada de $25 \times 25 \mathrm{~cm}$. Feringán no ha dejado testimonio gráfico de la disposición en planta de las estacas que forman el pilotaje de los cajones del muelle. Las representaciones del ingeniero se ocupan siempre de la sección transversal de éste $\mathrm{y}$ en la cuantiosa documentación manuscrita consultada tampoco hay una referencia explícita sobre este asunto. Sin embargo, esta investigación establece una disposición a tresbolillo para los pilotes del muelle, en base a la documentación manuscrita consultada.

Existen dos órdenes de estacas; una primera familia que se sitúa en los vértices de la cuadrícula que compondrá el enrejado de madera que se arriostra sobre ellos, y un segundo orden que se hinca en los centros de las celdas cuadrangulares que forman las vigas ortogonales con las que se construye el emparrillado.

Por tanto, existen dos familias de pilotes; aquellos que se sitúan en los vértices de la cuadrícula que compondrá el enrejado de madera que se arriostra sobre ellos y aquellos que se hincan en los centros de las celdas cuadrangulares que forman las vigas ortogonales con las que se construye este emparrillado.

Una vez definida la posición en planta de los pilotes se plantean dos preguntas al respecto: (1) ¿existen referencias anteriores sobre esta solución adoptada para la realización de la cimentación por pilotaje? y (2) ¿qué función tiene el pilote centrado en las celdas del enrejado?.
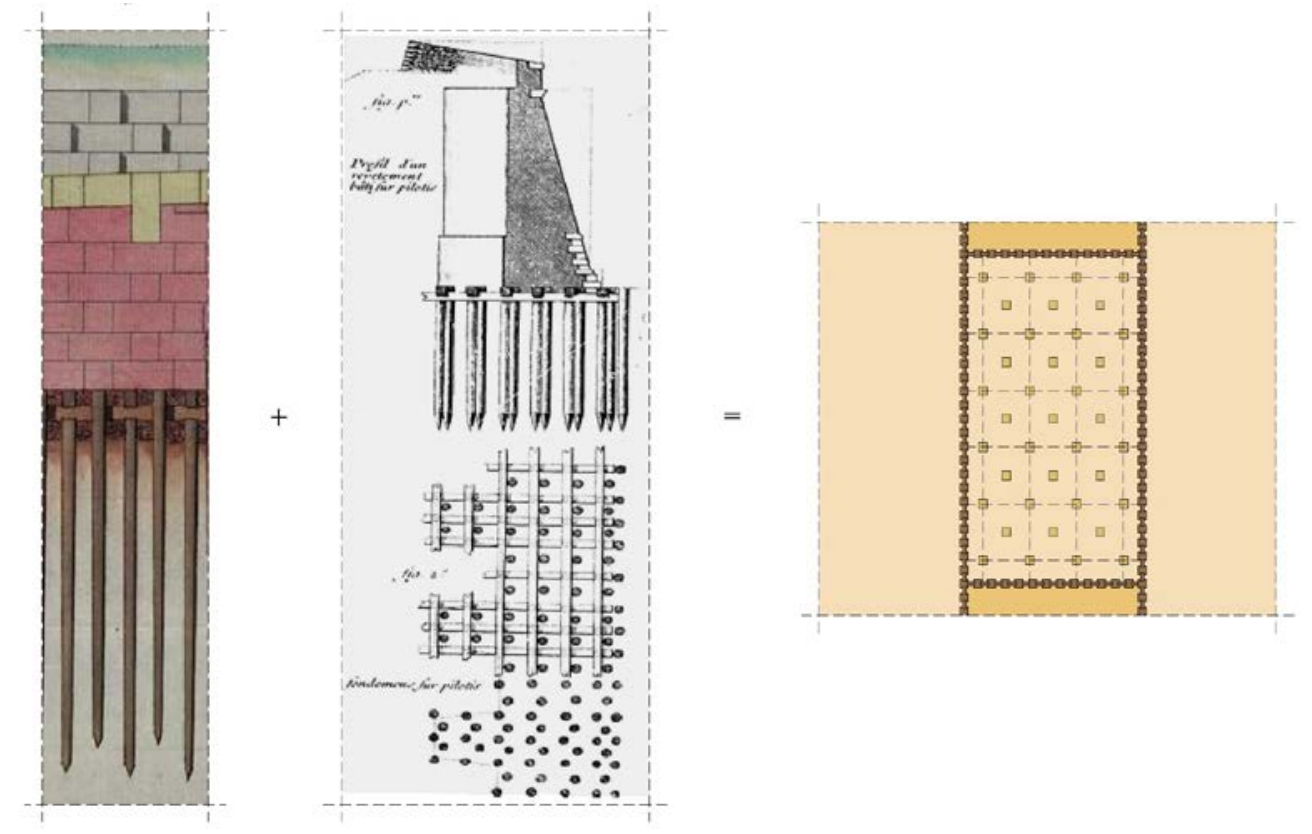

Fig. 6- Distribución en planta de los pilotes del cimiento del muelle de Cartagena en relación a la propuesta de Belidor para pilotar una muralla. (Peñalver, 2012)

La respuesta a la primera de ellas, se encuentra en el tratado de Belidor (1729), donde se propone como solución cuando se debe recurrir al pilotaje, la distribución de pilotes recogida en la imagen anterior. El ingeniero francés recomienda clavar unas estacas pasantes 
centradas en las celdas del enrejado pero tangentes a una de las "varengas" que la configuran. A la luz de la sección representada en su tratado este pilote asume la función de elemento estabilizador frente al vuelco del cimiento. Hay que pensar que Belidor representa en su dibujo, la sección de una muralla y propone clavar estos pilotes hacia el lado del foso de forma que, haciéndolo solidario al enrejado, impida el vencimiento de la muralla hacia esta excavación.

Aunque similar en sus planteamientos, los pilotes situados en el centro geométrico de los cuadros del enrejado de Feringán, no pueden cumplir esta función al estar completamente desolidarizados de la retícula. Entonces, ¿por qué Feringán no los obvia con el consiguiente ahorro de recursos que esto supondría para las obras?.

La respuesta a esta pregunta, se fue perfilando en el tiempo a través de la continua revisión y maduración de la documentación que se analiza durante el proceso de investigación. La colocación de la estaca central, no tiene otra función que servir como apoyo a los sillares que sentarán sobre el enrejado macizado. El aparejo de los sillares empleado en la construcción de la fábrica de sillería del muelle requiere de la colocación de este pilote para conseguir, como menciona Jorge Juan, que todas las piedras apoyen sobre pilotes.

[...] desde el qual se levanta el muelle de sillares de 8 pies de largo y 2 de ancho $y$ gruesso (procurando que todos apoyen sobre estaca) hasta 3 pies sobre el Nivel del mar. [...]

El enrejado fabricado por Feringán sobre las cabezas de los pilotes se configura mediante vigas endentadas de madera, que se cruzan ortogonalmente sobre las cabezas de los pilotes. Estas vigas se colocan en dos niveles diferentes: las inferiores, que apoyan directamente sobre las cabezas de las estacas de cimentación y que reciben el nombre de "cabezales", y las ortogonales a éstas que se cruzan por encima y que descansan sobre las primeras, recibiendo el nombre de "varengas" (Fig. 3).
La geometría endentada de estas vigas es de gran importancia para garantizar la unidad del conjunto e indispensable para el correcto funcionamiento del sistema. Aunque el macizado posterior del enrejado colabora en la unidad del conjunto, es la sección longitudinal en diente de sierra de estas vigas, la que cumple dos misiones fundamentales encaminadas a conseguir la necesaria rigidez del conjunto y que compensan el mayor coste en el labrado de la madera. Por un lado, permiten disminuir el espesor resultante del cruce de las vigas sobre las cabezas de los pilotes con lo que ello supone para el correcto clavado de los elementos y la estabilidad del nudo al descender su centro de gravedad. Por otro, garantizan el encastramiento de las piezas que se intersectan -estacas, cabezales y varengas-, no confiando la inmovilidad del conjunto únicamente a la cabilla de roble que las clava.

En este sentido, Feringán da un paso más en la solución propuesta por Belidor, en la que el francés sólo considera el endentado para las varengas dejando los cabezales de sección constante.

En cuanto a la modulación de la retícula que compone el enrejado de cimentación, se constituye mediante la adición de celdas cuadradas de 5 palmos (1,04 m). De esta manera se garantiza el apoyo de todos los sillares de la primera hilada del cimiento en al menos la cabeza de una estaca, tal y como explicaba Jorge Juan.

\subsection{La configuración del plano de apoyo. El macizado}

La última fase a acometer para finalizar la cimentación de pilotes es proceder al macizado del conjunto del emparrillado. Será necesario garantizar la correcta compactación del material de relleno, tanto en el espacio bajo el enrejado hasta el nivel del terreno natural como en las celdas del emparrillado, hasta conseguir un plano horizontal nivelado con la cara superior de las varengas. La colocación de este material debe garantizar el envolvimiento correcto de las cabezas de los pilotes que afloran a la superficie. 
El mortero que se emplea para este relleno está compuesto por piedra menuda, cal y puzolana. Su puesta en obra queda perfectamente descrita por Jorge Juan en las siguientes líneas.

[...]. Tanto los huecos que dexa este enrrejado, como lo que está debajo de Varengas y Cabezales se llena de Piedra menuda, Cal y Porcelana, macizando y apretando el todo a fuerza de Pilon de hierro, poniendo mas y mas material hasta hacer un Plan horizontal con lo mas alto de las Varengas: desde el cual se levanta el Muelle de sillares [...].

\section{Conclusiones}

Tras la investigación realizada sobre la construcción del muelle de la dársena del Puerto de Cartagena durante el siglo XVIII y en lo relativo a su cimentación por pilotaje, podemos concluir que:

1. Las obras que se realizan para la construcción de la dársena de Cartagena son un reto para la técnica existente en el momento de su materialización y no fruto de ella.

La decisión de construir una dársena para el arsenal de Cartagena se adopta por motivos estratégicos fruto de la renovada política naval borbónica y será necesario que la técnica evolucione para poder materializar tal decisión. Se requerirá del perfeccionamiento de los sistemas constructivos conocidos, así como de los medios auxiliares existentes para su realización (tablestacados, bombas, cabrestantes y martinetes). La correcta planificación de los procesos de puesta en obra de estas singulares soluciones constructivas, posibilitará realizar la construcción de esta dársena artificial con los excepcionales condicionantes impuestos por la cercanía del Mediterráneo.

2. La naturaleza del terreno encontrado y el nivel freático que se detecta tras la excavación, determinan y modifican las soluciones constructivas planteadas inicialmente para cimentar el muelle, así como las técnicas de puesta en obra previstas en origen.

3. Los condicionantes técnicos y funcionales que determinan la tipología de cimiento a construir en el muelle, son las aguas que afluyen a las excavaciones y el calado necesario para la dársena.

4. El tablestacado utilizado como estructura de contención en la excavación, es también considerado como parte integrante del cimiento del muelle.

5. Los subsistemas que configuran la cimentación profunda realizada en el muelle son tres: el tablestacado o "palplanchado", los pilotes o "estacas" y el enrejado o “embarengado”.

6. La pilotes se disponen a tresbolillo para garantizar que todos los sillares apoyan en al menos una cabeza de estaca.

7. La rigidez del enrejado que se construye sobre las cabezas de los pilotes a modo de encepado, se consigue gracias a la geometría endentada de las vigas de madera que lo componen y el macizado de sus huecos.

\section{Notas}

(1) A.G.S., Marina, leg. 376. Carta de D. Sebastián Feringán al marqués de la Ensenada informar sobre el estado de las obras y solicitando mayor número de ingenieros a sus órdenes, 27/01/1750.

(2) A.G.S., Marina, leg. 376. Carta de D. Sebastián Feringán al marqués de la Ensenada informando sobre su opinión si los veneros que aparecen bajo los cimientos pueden perjudicar a la solidez de los muelles y describe la construcción de éstos, 27/07/1751.

(3) Idem.

(4) B.N., Mss. 3.372-76. Los Veinte y un Libros de los Ingenios y Máquinas. Tomo Quinto, Libro 19, p. 59. [Juanelo Turriano], s. XVI.

(5) A.G.S., Marina, leg. 376. Carta de D. Sebastián Feringán Cortés al marqués de la Ensenada informando sobre el estado de las obras, 24/06/1750.

(6) A.G.S., Marina, leg. 376. Carta de D. Jorge Juan y Santacilia al marqués de la Ensenada contestando a la consulta sobre la firmeza del terreno para fundar los muelles y edificios del Arsenal de Cartagena, 03/08/1751. 
(7) A.G.S., Marina, leg. 376. Carta de D. Jorge Juan y Santacilia al marqués de la Ensenada contestando a la consulta sobre la firmeza del terreno para fundar los muelles y edificios del Arsenal de Cartagena, 03/08/1751.

\section{Referencias}

Belidor, B. F. (1729). La Science des ingénieurs dans la conduicte des travaux de fortification et d'architecture civile dedié au Roy. Claude Jombert Ed. París.

Peñalver Martínez, M.J. (2012). Génesis y materialización de la dársena del puerto de Cartagena a lo largo del s. XVIII. Una propuesta metodológica para el análisis arquitectónico del patrimonio construido. Tesis doctoral, UPCT-ETSAE, Cartagena, p. 450.

VV.AA. (1985). Máquinas y artes de construcción portuaria en la exposición de puertos y fortificaciones en América y Filipinas. Ministerio de Fomento, CEHOPU. Madrid. Pp. 11-14. 